\title{
COMPACTAÇÃO DE MISTURAS SOLO-GRITS PARA EMPREGO EM ESTRADAS FLORESTAIS: INFLUÊNCIA DO TEMPO DECORRIDO ENTRE MISTURA E COMPACTAÇÃO NA RESISTÊNCIA MECÂNICA ${ }^{1}$
}

\author{
Reginaldo Sérgio Pereira², Carlos Cardoso Machado ${ }^{3}$ e Dario Cardoso de Lima ${ }^{4}$
}

\begin{abstract}
RESUMO - Vários fatores influenciam a execução e a "performance" de camadas de pavimentos rodoviários constituídas de solos estabilizados quimicamente, com destaque para a homogeneização da mistura e o período de tempo decorrido entre a mistura e a compactação, bem como para a escolha do equipamento de compactação. Aborda-se, no presente artigo, a influência do tempo decorrido entre a mistura e a compactação (TMC) nos parâmetros de compactação e na resistência mecânica de misturas de dois solos típicos da Zona da Mata Norte de Minas Gerais, com o resíduo da indústria de celulose denominado "grits", com vistas à sua aplicação em estradas florestais. Analisa-se, também, a influência de diferentes lotes desse resíduo na resistência mecânica das misturas. Trabalhou-se com os resultados de ensaios de índice de suporte Califórnia (ISC ou CBR) realizados em corpos-de-prova de misturas moldados na energia de compactação do ensaio Proctor Intermediário. Os resultados permitem concluir que o tempo decorrido entre mistura e compactação influencia significativamente a resistência mecânica das misturas analisadas, recomendando-se, para fins práticos, que estudos dessa natureza devam ser feitos em bases regionais de ocorrência de solos. Observou-se, também, influência significativa do lote na resistência mecânica das misturas.
\end{abstract}

Palavras-chave: Estabilização de solos, "grits”, tempo entre mistura e compactação e estradas florestais.

\section{COMPACTION OF SOIL-GRITS MIXTURES FOR USE IN FORESTRY ROAD: INFLUENCE OF TIME BETWEEN MIXTURE AND COMPACTION ON MECHANICAL STRENGTH}

\begin{abstract}
Many factors affect the construction and performance of chemically stabilized highway pavement soil layers, especially mixture homogenization, time between mixture and compaction, and selection of compaction equipment. Regarding forest road application, this paper addresses the influence of time between mixture and compaction (TMC) on compaction optimum parameters and on mechanical strength of mixtures of two typical soils from the region 'Zona da Mata Norte', State of Minas Gerais, and grits, a waste from the cellulose industry, as well as the influence of grits sampling on the mechanical strength of soil 2-grits mixtures. California bearing ratio $(C B R)$ data from laboratory specimens compacted at the Intermediate Proctor compaction effort were used throughout the study. The laboratory testing data supported that time between mixture and compaction significantly affected the mechanical strength of the tested soil mixtures. Regarding soil samples and from a practical point of view, it is recommended that such studies should be always performed on regional basis. It was observed significant influence of grits sampling on the mechanical strength of soil 2-grits mixtures.
\end{abstract}

Keywords: Soil stabilization, grits, time between mixture and compaction, forest roads.

\footnotetext{
${ }^{1}$ Recebido em 22.11.2005 e aceito para publicação em 05.04.2006.

${ }^{2}$ Programa de Pós-Graduação em Ciências Florestais da UFV. E-mail: <reginaldosp1 @ yahoo.com.br>.

${ }_{3}^{3}$ Departamento de Engenharia Florestal da UFV, Viçosa-MG, Brasil. E-mail: <machado@ufv.br>.

${ }^{4}$ Departamento de Engenharia Civil da UFV, Viçosa-MG, Brasil. E-mail: <declima@ufv.br>.
} 


\section{INTRODUÇÃO}

No processo construtivo de estradas, a compactação de camadas de solos estabilizados quimicamente constitui-se em uma etapa de grande relevância para o bom desempenho da obra. Dentre os aspectos de interesse na "performance" das misturas, pode-se referir a: tipos de solo e estabilizante, teor de umidade da mistura, processo de mistura, tempo decorrido entre mistura e compactação, tipo de equipamento e energia empregada no processo de compactação e período de cura da camada estabilizada.

Em se tratando de compactação de solos realizada em laboratório, a normatização técnica da Associação Brasileira de Normas Técnicas (ABNT, 1986) prescreve que, após a adição de água às amostras a serem submetidas à compactação, deve-se observar um tempo mínimo de 24 horas antes da compactação. Em especial, para solos argilosos, essa exigência visa possibilitar melhor homogeneização da água na amostra de solo. No entanto, ao se empregarem as técnicas de estabilização química de solos, este é um aspecto de importância significativa para a boa qualidade mecânica das misturas, pois, se na mistura já ocorreram reações de cimentação de curto prazo antes da compactação, certamente parte do estabilizante adicionado ao solo foi utilizada na sua confecção. Considerando-se que essas ligações podem ser quebradas no processo de compactação da camada estabilizada, reduz-se, assim, o quantitativo de estabilizante disponível para a cimentação das partículas de solo, com conseqüente queda na resistência mecânica da mistura. Nesse contexto, ganha importância o tempo decorrido entre mistura e compactação de camadas estabilizadas quimicamente.

Com relação ao tópico em questão, refere-se que na execução de camadas de misturas solo-cimento é comum exigir que o tempo decorrido entre mistura e final de compactação não exceda 3 horas (SENÇO, 2002), bem como que resultados de pesquisas indicam que dois solos geotecnicamente classificados como residuais maduro e jovem apresentam tempos ótimos entre mistura e compactação, respectivamente, de 0 e 4 horas, quando estabilizados com o produto RBI Grade 81 (TRINDADE, et al., 2005) e escória de alto-forno granulada moída (SANT'ANA, 2003). Em misturas solo-cal, Dantas (1970) apud Guimarães (1971) evidenciaram que igual compactação imediatamente e decorridas 24 horas após a execução da mistura levou à redução da densidade e da resistência. Certamente, essas constatações indicam que estudos sobre esse tópico devem ser desenvolvidos, considerando-se a gênese e os processos de formação dos solos, adquirindo, pois, um caráter regional.

Como parte integrante de uma série de estudos sobre a viabilidade de emprego do resíduo "grits" na construção de estradas florestais, o presente artigo objetivou analisar a influência do tempo decorrido entre mistura e compactação de combinações solo-grits nas suas resistências mecânicas, através de um programa de ensaios geotécnicos de laboratório.

\section{MATERIAL E MÉTODOS}

\subsection{Material}

Fez-se uso do resíduo “grits", gerado no processo de recuperação da soda cáustica, na etapa de cozimento de cavacos de madeira de eucalipto, para produção de celulose. O interesse se deveu aos constituintes químicos presentes em tal material, em especial o $\mathrm{CaO}$ (óxido de cálcio), que demonstram potencial para a estabilização química de solos para construção de estradas, principalmente as florestais, que se situam a distâncias de transporte relativamente pequenas do local de produção e coleta do resíduo.

Trabalhou-se com duas amostras de solos típicos da Zona da Mata Norte de Minas Gerais, coletadas na microrregião da cidade de Viçosa, caracterizadas geotecnicamente e utilizadas em estudos anteriores envolvendo misturas com outros estabilizantes químicos, que podem ser descritas como se segue: (i) solo 1 Geotecnicamente, trata-se de um solo residual maduro de gnaisse de comportamento laterítico, pedologicamente classificado como Latossolo Vermelho-Amarelo, laterítico, de textura argilo-areno-siltosa, cuja amostra foi coletada no horizonte $\mathrm{B}$ ( $\cong 6 \mathrm{~m}$ de profundidade) de um talude de corte situado na rodovia que liga Viçosa a Paula Cândido, nas proximidades da Estação de Tratamento de Água de Viçosa; e (ii) solo 2 Geotecnicamente, trata-se de um solo residual jovem de gnaisse, de comportamento não-laterítico, de textura areno-argilo-siltosa, cuja amostra foi coletada no horizonte

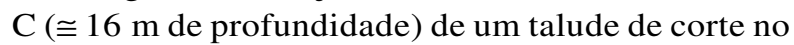
Campus da Universidade Federal de Viçosa (UFV), na Vila Secundino. O Quadro 1 apresenta a caracterização geotécnica, bem como a classificação pelo Sistema TRB e pela Metodologia MCT, das amostras de solo empregadas. 
Quadro 1 - Caracterização geotécnica e classificação das amostras de solo Table 1 - Geotechnical characterization and classification of soil samples

\begin{tabular}{lccccccc}
\hline Solos & \multicolumn{2}{c}{ Granulometria (ABNT, 1986) } & \multicolumn{2}{c}{ Plasticidade } & \multicolumn{2}{c}{ Peso Esp. Sól. } & Classificação \\
& \%Areia & \%Silte & \%Argila & LL\% & IP\% & TRB $\left(\right.$ KN/m $\left.{ }^{3}\right)$ & MCT \\
\hline 1 & 28 & 12 & 60 & 75 & 29 & 27,63 & A-7-5 (20) \\
2 & 74 & 11 & 15 & 27 & 10 & LG' \\
\hline
\end{tabular}

\subsection{Métodos}

\subsubsection{Quantitativos de "grits"}

O resíduo "grits" foi utilizado nos seguintes quantitativos: $4,8,12,16,20,24$ e $28 \%$, porcentuais esses calculados sobre a massa de solo seco.

\subsubsection{Períodos de tempos decorridos entre mistura e compactação em laboratório (TMC)}

As misturas solo-grits foram executadas da seguinte forma: (i) adição do resíduo ao solo; (ii) homogeneização manual das misturas seguida por peneiramento delas na peneira de abertura nominal $4,8 \mathrm{~mm}$; (iii) adição de água às misturas de solo-grits seguida por peneiramento delas na peneira de abertura nominal de $4,8 \mathrm{~mm}$; e (iv) acondicionamento da mistura em sacos plásticos para preservação dos seus teores de umidade. Após essa etapa, respeitaram-se tempos de descanso entre mistura e compactação dos corpos-de-prova (TMC), conforme se segue: (i) etapa 1 - Tempos extremos: 0 (compactação imediata), 24 e 72 horas; e (ii) etapa 2 - Tempos de situações práticas de campo de 2, 4, 6 horas.

\subsubsection{Avaliação da resistência mecânica}

Para avaliar a resistência das misturas solo-grits, fez-se uso do ensaio CBR normatizado pelo DNIT (1994) ME 129. Trabalhou-se na energia de compactação do ensaio Proctor intermediário.

\section{RESULTADOS E DISCUSSÃO}

\subsection{Influência do TMC na densidade das misturas solo-grits compactadas}

A influência do TMC na densidade das misturas solo-grits compactadas é ilustrada na Figura 1, observando-se que: (i) em se tratando das misturas solo 1-grits, não há tendência comum para os valores de peso específico seco máximo $\left(\gamma_{\text {dmáx }}\right)$; em todos os teores de "grits", o $\gamma_{\text {dmáx }}$ decresceu bruscamente para teores de resíduo até $12 \%$, em função do aumento do TMC, sendo notados pequena queda no traço de $16 \%$ de resíduo e aumentos desse parâmetro para os teores de 20 e $24 \%$, provavelmente devido à maior incorporação no solo 1 de partículas mais grosseiras, oriundas do resíduo; e (ii) nas misturas solo 2-“grits”, notaram-se, em todos os tratamentos, maiores valores de $\mathrm{g}_{\text {dmáx }}$ nas misturas compactadas imediatamente após a adição do resíduo aos solos, evidenciando tendência a maiores valores de resistência para esse TMC.

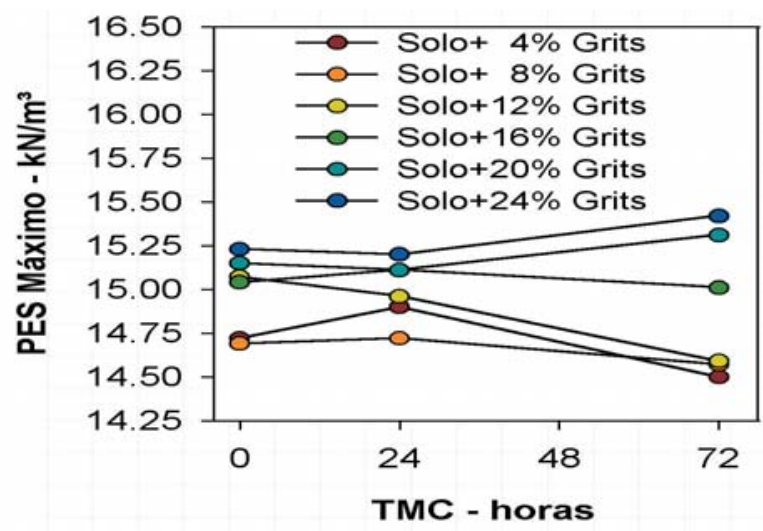

a) Misturas Solo1-Grits

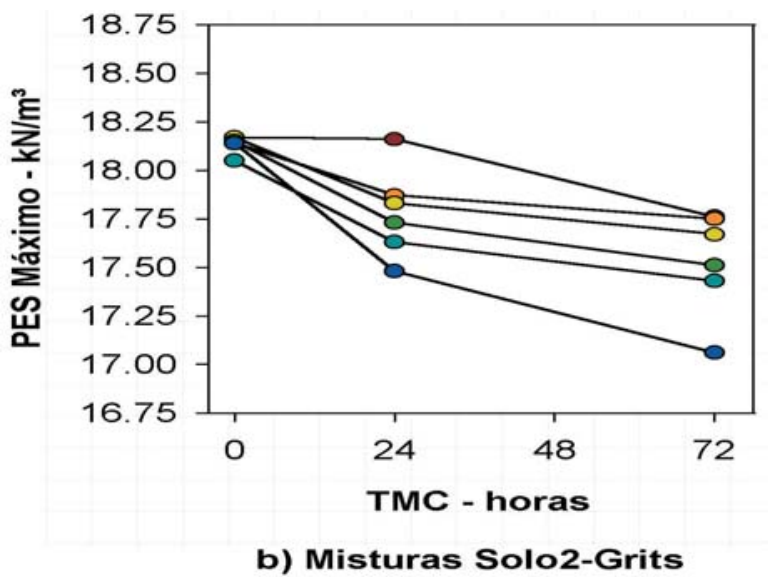

Figura 1 - Influência do tempo entre mistura e compactação (TMC) na densidade das misturas solo-"grits" compactadas.

Figure 1 - Influence of time between mixture and compaction (TMC) on the density soil-grits mixtures compacted.

R. Árvore, Viçosa-MG, v.30, n.3, p.421-427, 2006 


\subsection{Influência do TMC na resistência mecânica das misturas - etapa 1: efeito de TMCs extremos de mistura}

A Figura 2 ilustra o comportamento mecânico das misturas solo-grits compactadas, diante dos TMC de 0,24 e 72 horas, tomando por base os resultados de ensaios CBR. A análise dos dados apresentados nessa figura pode ser assim: (i) misturas solo 1-grits - Observouse pouca influência do TMC nos valores CBR em teores de resíduo na faixa de 0 a $16 \%$ e que ocorreram variações nos valores CBR para os teores de resíduo entre 16 e $28 \%$, com decréscimos pequenos para o TMC de 24 horas e acréscimos significativos para o TMC de
72 horas, em especial na mistura com $24 \%$ de resíduo (ganho de 60\%); verificou-se pouca influência do TMC no parâmetro expansão medida no ensaio CBR, havendo tendência para a ocorrência de maiores e menores valores, respectivamente, para TMC de 0 e 72 horas; e (ii) misturas solo 2- "grits" - Para valores de resíduo na faixa de 8 a $28 \%$, observou-se que, aumentando o TMC, há tendência nítida de decréscimo nos valores CBR, atingindo quedas da ordem de 47 e $56 \%$, respectivamente, para TMCs de 24 e 72 horas e para o teor de resíduo de 24\%; com relação à expansão, ocorreu tendência a acréscimos, com aumentos no TMC para teores de resíduo entre 12 e $28 \%$.

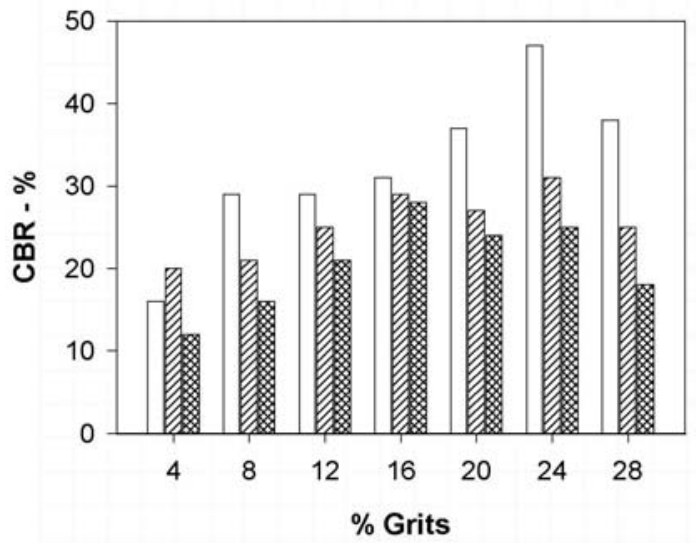

b) Capacidade Suporte Misturas Solo2-Grits

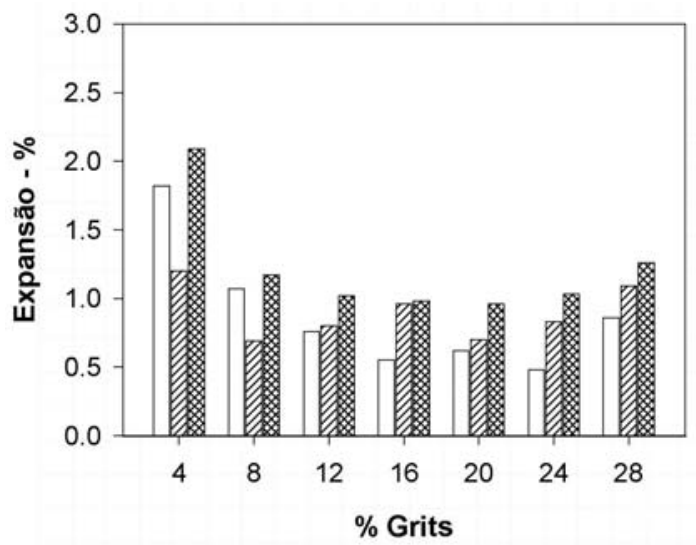

d) Expansão Misturas Solo2-Grits

Figura 2 - Influência do TMC no CBR e expansão das misturas solo-"grits".

Figure 2-Influence of TMC on CBR and swelling for soil-grits mixtures.

R. Árvore, Viçosa-MG, v.30, n.3, p.421-427, 2006 
Aspectos práticos da análise dos resultados apresentados na Figura 2 são como se segue: (i) com relação ao solo 1, de textura argilosa, observou-se que valores maiores de TMC levaram a aumentos no parâmetro CBR das misturas, provavelmente devido ao fato de que, para esses TMCs, ocorreram, majoritariamente, reações de troca catiônica que foram responsáveis por mudanças estruturais e texturais na massa de solo, não ocorrendo, para fins práticos, a efetivação de ligações de cimentação entre partículas do solo; e (ii) no que tange ao solo 2, de textura arenosa, com o aumento do TMC verificou-se a efetivação de um número maior de ligações de cimentação entre partículas que, quando da compactação, são quebradas, resultando, pois, em quedas nos valores CBR e, conseqüentemente, na resistência mecânica das misturas.

\subsection{Influência do TMC na resistência mecânica das misturas - etapa 2: efeito de TMCs que refletem situações de compactação no campo}

A Figura 3 ilustra o comportamento mecânico das misturas solo-grits, para TMCs representativos de situações práticas de campo. Para as misturas solo 1-“grits”, variações no TMC foram responsáveis por oscilações em seus valores de resistência mecânica, não se identificando, porém, uma tendência para se identificar um valor ótimo, podendose citar, por exemplo, os teores de 24 e $28 \%$ de resíduo que apresentaram maiores valores $\mathrm{CBR}$ para o $\mathrm{TMC}$ de 4 horas.

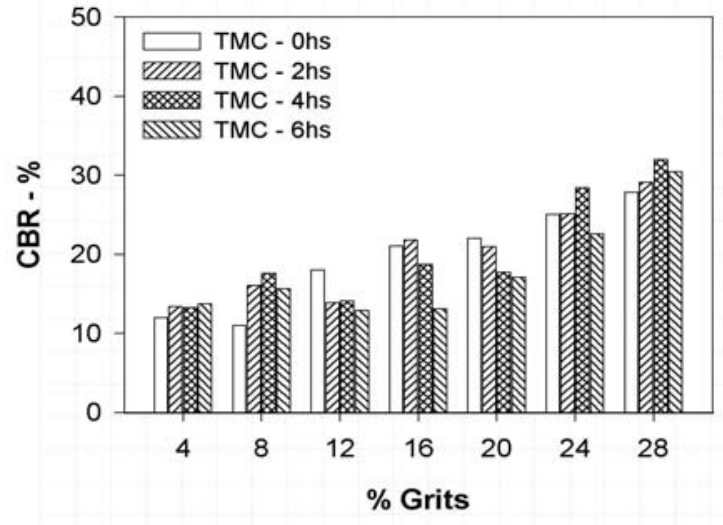

a) Capacidade Suporte Misturas Solo1-Grits

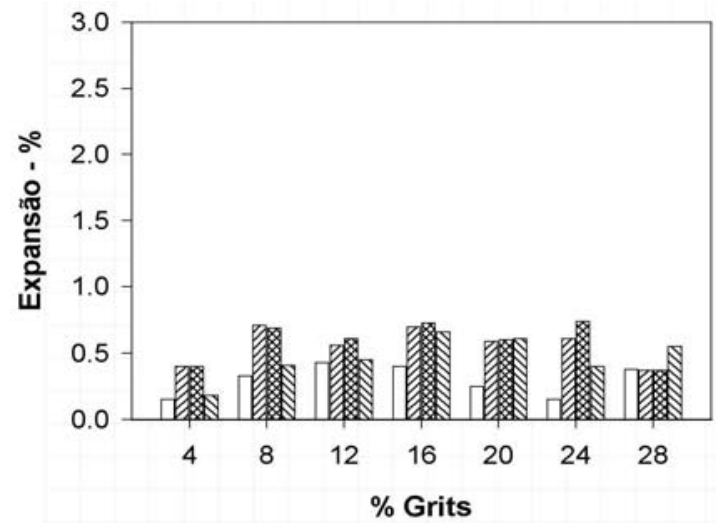

c) Expansão Misturas Solo1-Grits

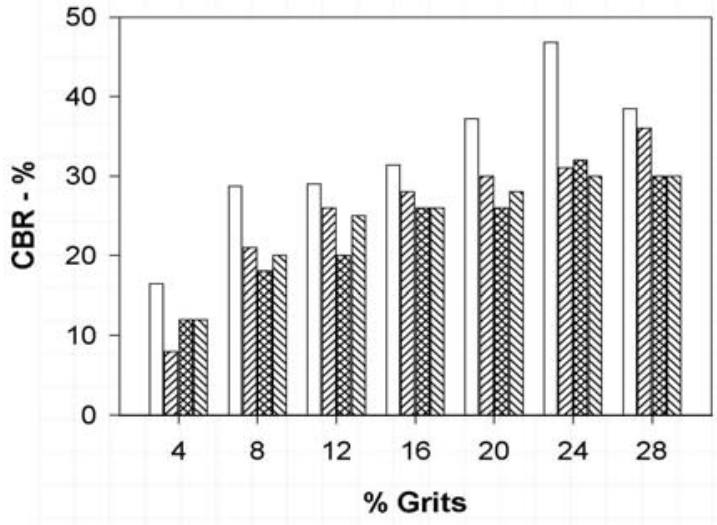

b) Capacidade Suporte Misturas Solo2-Grits

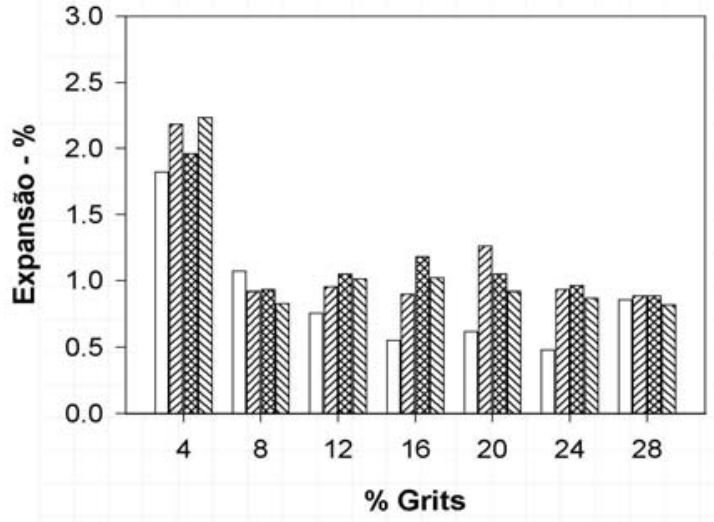

d) Expansão Misturas Solo2-Grits

Figura 3 - CBR e expansão das misturas solo-"grits", considerando-se TMCs que refletem situações de campo. Figure 3-CBR and swelling of soil-grits mixtures regarding TMC applied to field conditions. 
Com relação à expansão, considerado um parâmetro que pode exercer efeito deletério no comportamento mecânico das misturas, a compactação imediatamente após a mistura proporcionou o menor valor e, portanto, a melhor resposta em termos de comportamento mecânico. Para as misturas solo 2-“grits”, a compactação imediata continuou proporcionando maiores valores de CBR, em todos os traços de resíduo considerados, e menores valores de expansão para alguns tratamentos.

\subsection{Efeito do lote de resíduo na resistência mecânica das misturas}

Ao se trabalhar com resíduos industriais, uma preocupação que se deve ter é com a utilização de produtos que apresentem composições químicas similares, de modo a se produzirem misturas com mesmas respostas mecânicas, para fins de engenharia. Assim, buscou-se no presente trabalho analisar a influência do lote do resíduo na resistência mecânica das misturas do solo 2, trabalhando-se com dois lotes, aqui denominados 1 e 2 . As características químicas desses lotes são apresentadas no Quadro 2, em que se atenta para os teores de sódio e óxido de cálcio, e os resultados desse estudo são apresentados na Figura 4.

A análise dos dados apresentados na Figura 4 possibilita inferir que o resíduo proveniente do lote 1 apresentou maior potencialidade como estabilizante do que o resíduo do lote 2, visto que, em todas as misturas e TMCs considerados, foi possível atingir valores maiores de resistência mecânica e valores menores de expansão. Verificou-se que não houve tendência à maior discrepância no parâmetro CBR entre lotes de "grits", em função dos TMCs, destacando-se apenas os valores encontrados para os teores de 12, 16, 20 e $24 \%$ para o TMC de 24 horas, que apresentaram maior diferencial entre lotes.

A expansão apresentou maior discrepância entre lotes para o TMC de 72 horas, em todos os teores de resíduo. Essa diferença entre lotes é atribuída ao local de coleta do resíduo no aterro sanitário da empresa produtora, visto que, dependendo da posição e da época de coleta, que determina o tempo de exposição às intempéries climáticas, o resíduo pode sofrer alterações nas suas propriedades químicas.

Quadro 2 - Caracterização química do "grits" dos lotes 1 e 2

Table 2 - Characterization of grits from samples 1 and 2

\begin{tabular}{|c|c|c|c|c|c|c|c|c|c|}
\hline \multirow[t]{2}{*}{ Grits } & \multirow[t]{2}{*}{ Local da Coleta } & \multirow[t]{2}{*}{ Data } & \multicolumn{7}{|c|}{ Constituição Química } \\
\hline & & & $\mathrm{P}$ & $\mathrm{K}$ & $\mathrm{Na}$ & $\mathrm{CaO}$ & $\mathrm{MgO}$ & $S$ & Al \\
\hline Lote 1 & Pátio da Empresa & Out./00 & 0,26 & 0,12 & 0,70 & 53,50 & 0,60 & 0,18 & 0,09 \\
\hline Lote 2 & Aterro Sanitário & Jun./03 & 0,37 & 0,04 & 0,22 & 35,38 & 0,43 & 0,13 & 0,13 \\
\hline
\end{tabular}

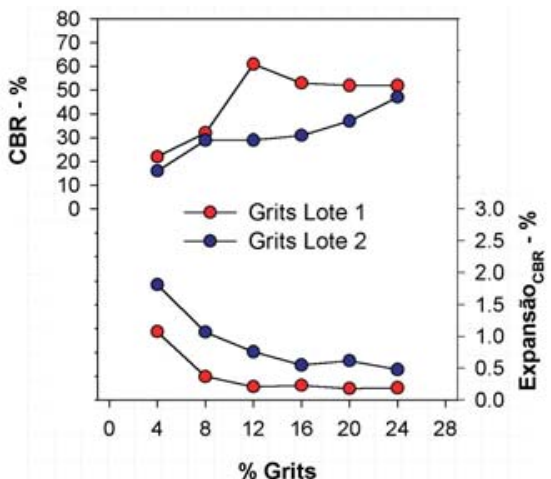

a) Compactação Imediata

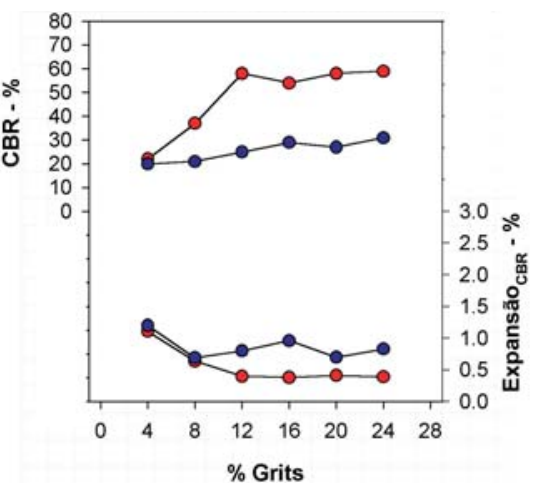

b) Compactação Decorridas 24 horas

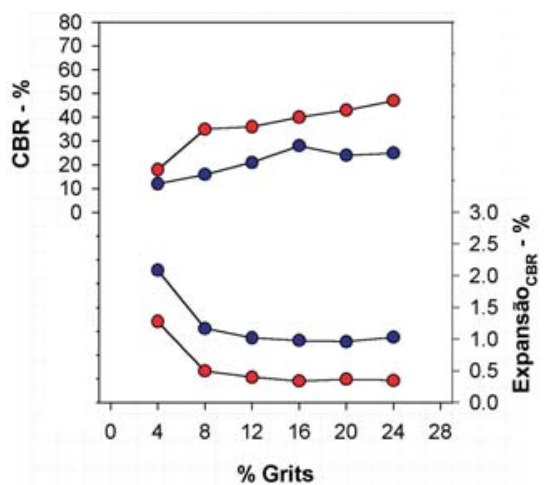

c) Compactação Decorridas 72 horas

Figura 4 - CBR e expansão das misturas solo 2-"grits" das diferentes TMCs, considerando-se os lotes 1 e 2 de "grits". Figure 4-CBR and swelling values of soil 2-grits mixtures regarding different TMC's and grits samples 1 and 2. 


\section{CONCLUSÕES}

Os resultados dos estudos de laboratório permitiram concluir que o tempo entre mistura e compactação influenciou significativamente o valor da resistência mecânica (CBR) e da expansão das misturas solo-grits. Nas misturas solo 1-“grits", essa influência foi menos significativa, não sendo possível identificar uma tendência para um TMC ótimo. Já nas misturas solo 2"grits", chegou-se a maiores valores de CBR e menores valores de expansão para as combinações compactadas imediatamente após a mistura. Ressalta-se que nessas misturas o lote do resíduo influenciou, também, os valores de resistência mecânica e expansão.

\section{AGRADECIMENTOS}

Ao CNPq e à FAPEMIG, pelo apoio financeiro ao projeto; à CAPES, pelo fornecimento da bolsa de doutorado; e à CENIBRA, pelo fornecimento do resíduo.

\section{REFERÊNCIAS BIBLIOGRÁFICAS}

\section{ASSOCIAÇÃO BRASILEIRA DE NORMAS} TÉCNICAS - ABNT. NBR 7181: solo: análise granulométrica. Rio de Janeiro: 1984. 13 p.
ASSOCIAÇÃO BRASILEIRA DE NORMAS

TÉCNICAS - ABNT. NBR 7182: solo: ensaio de compactação. Rio de Janeiro: 1986.10 p.

\section{DEPARTAMENTO NACIONALDE}

INFRAESTRUTURA DE TRANSPORTES - DNIT.

ME129: solos: compactação utilizando amostras não trabalhadas. Rio de Janeiro: 1994. 7 p.

GUIMARÃES, J.E.P. Estabilização de solos: nova e importante aplicação da cal, seus conceitos e suas vantagens. São Paulo: ABPC, 1971. 67 p. (Boletim, 5).

SANT'ANA, A.P. Caracterização tecnológica de misturas solo-escória de alto forno moída para fins rodoviários. 2003. 74f. Dissertação (Mestrado em Geotecnia)Universidade Federal de Viçosa, Viçosa, 2003.

SENÇO, W. Manual de técnicas de pavimentação. São Paulo: PINI, 2002. v. 2, 671 p.

TRINDADE, T. P. et al. Estabilização química do subleito de estradas florestais: influência do tempo decorrido entre a mistura e a compactação na resistência mecânica de misturas solo-RBI Grade 81 . Revista Árvore, v. 29, n. 3, p. 423-418, 2005. 